\title{
Kinematics of the dwarf irregular galaxy GR8
}

\author{
A. Begum and J. N. Chengalur ${ }^{\star}$ \\ National Centre for Radio Astrophysics, Post Bag 3, Ganeshkhind, Pune 411 007, India \\ Received 15 May 2003 / Accepted 22 July 2003

\begin{abstract}
We present deep, high velocity resolution $\left(\sim 1.6 \mathrm{~km} \mathrm{~s}^{-1}\right)$ Giant Meterwave Radio Telescope HI $21 \mathrm{~cm}$ synthesis images for the faint $\left(M_{B} \sim-12.1\right)$ dwarf irregular galaxy GR8. We find that the velocity field of the galaxy shows a clear systematic large scale pattern, with a maximum amplitude $\sim 10 \mathrm{~km} \mathrm{~s}^{-1}$. Neither pure rotation, nor pure radial motion alone can fit the observed velocity field; however a combination of radial and circular motions can provide a reasonable fit. The most natural interpretation is that the neutral ISM, in addition to rotating about the center, is also expanding outwards, as a result of energy input from the ongoing star formation in the galaxy. Support for this interpretation comes from the fact that the pressure in the HII regions in the galaxy is known to be substantially ( $\sim 55$ times) higher than the average pressure in the gas disk. It is, however, also possible that the velocity field is the result of the gas swirling inwards, in which case GR8 could be in the process of formation via the merger of subgalactic clumps.
\end{abstract}

Key words. galaxies: evolution - galaxies: dwarf - galaxies: kinematics and dynamics galaxies: individual: GR8 radio lines: galaxies

\section{Introduction}

Although bright irregular galaxies have rotating gas disks, it is unclear whether the faintest dwarf irregular galaxies are rotationally supported or not. Côté et al. (2000), based on a study of the kinematics of eight dwarf irregular galaxies (with magnitudes varying from $M_{B}=-16.7$ to $M_{B}=-11.3$ ) suggest that normal rotation is seen only in dwarfs brighter than $M_{B} \sim-14$. This is consistent with the earlier findings of Lo et al. (1993), who, based on an interferometric study of faint dwarf galaxies (with $M_{B} \sim-9$ to $M_{B} \sim-15$ ) found that only two of their sample of nine galaxies showed ordered velocity fields. However, this conclusion has been questioned by Skillman (1996) who pointed out that the interferometric observations of Lo et al. (1993) lacked sensitivity to low extended HI distribution and could thus have been insensitive to the large scale velocity field. Further, Begum et al. (2003) showed that the dwarf irregular galaxy Camelopardalis B, despite being extremely faint $\left(M_{B} \sim-10.9\right)$ nonetheless has a regular velocity field, consistent with that expected from a rotating disk. So, some faint galaxies at least, have rotating HI disks. What about the rest? If gas in faint dwarf galaxies is not supported by rotation, what provides the energy that keeps it from collapse? For very faint dwarf irregular galaxies, the binding energy of the gas is not much larger than the energy output of a few supernovae. Star formation in such galaxies could hence have a profound effect on the kinematics of the ISM. Indeed, the faintest dwarf

Send offprint requests to: A. Begum,

e-mail: ayesha@ncra.tifr.res.in

* e-mail: chengalur@ncra.tifr.res.in galaxies are expected to lose a substantial part of their gas due to the energy deposited in the ISM by supernovae from the first burst of star formation (e.g. Dekel \& Silk 1986). Some observational support for such models is provided by the large expanding HI supershells seen in the ISM of some dwarf irregular galaxies with active star formation (e.g. Ott et al. 2001). In this paper we discuss the issue of the kinematics of faint dwarf galaxies, and its possible connections with energy input from stellar processes, in the specific context of the faint $\left(M_{B} \sim-12.1\right)$ dwarf irregular galaxy GR8.

GR8 was first discovered by Reaves (1956) in the course of a survey for dwarf galaxies in the direction of the Virgo Cluster. It has also been cataloged as DDO 155 by van den Bergh (1959). The original distance estimates for GR8 were in the range 1.0-1.4 Mpc (Hodge 1967; De Vaucouleurs \& Moss 1983; Hoessel \& Danielson 1983), which would make GR8 a probable member of the local group. However, recent estimates give somewhat larger distances. Tolstoy et al. (1995) estimated a distance of $2.2 \mathrm{Mpc}$ (based on observations of the only detected Cepheid variable). This estimate is in excellent agreement with that of Dohm-Palmer et al. (1998) which is based on the brightness of the tip of the red giant branch. From the location for the local group barycenter given by Courteau \& van den Bergh (1999) one can compute that this distance places GR8 well outside the local group zero velocity surface. Consistent with this, van den Bergh (2000) does not classify GR8 as a member of the local group.

GR8 has a patchy appearance in optical images, with the emission being dominated by bright blue knots. H- $\alpha$ imaging (Hodge 1967) shows that these knots are sites of active 
star formation. However, in addition to the bright blue knots, GR8 also possesses faint extended emission (Hodge 1967; De Vaucouleurs \& Moss 1983), indicative of earlier episodes of star formation. Indeed, CM diagrams (based on HST imaging, Dohm-Palmer et al. 1998), show that although the bright star forming knots in GR8 have stars which are younger than $\sim 10 \mathrm{Myr}$, the galaxy also contains stars which are older than a few Gyr. Despite this long history of star formation, the metallicity of the star forming knots in GR8 is extremely low, 3\% solar (Skillman et al. 1988b). This makes it one of the lowest metallicity galaxies known (Kunth \& Östlin 2000). In keeping with this low metallicity, despite the fact that the star forming regions are expected to be associated with molecular gas, CO has not been detected in the galaxy (Verter \& Hodge 1995).

There have been two independent studies of the kinematics of HI in GR8, both using the VLA. However these two studies resulted in very different interpretations of the galaxy's kinematics. Carignan et al. (1990) assumed that the observed velocity field was produced by rotation and used it to derive a rotation curve. On the other hand Lo et al. (1993) interpreted the velocity field as being due to radial motions (i.e. either expansion or contraction). Both of these studies were based on modest $\left(\sim 6 \mathrm{~km} \mathrm{~s}^{-1}\right)$ velocity resolution observations. Further both observations used the VLA $\mathrm{C}$ array, and hence were not sensitive to emission from the extended low surface brightness portions of the HI disk. There has also been a recent high velocity resolution VLA (Cs array) based study of GR8 (Young et al. 2003). This study was focused on the local connections between the ISM and star formation and not the large scale kinematics of the gas. Although Young et al. (2003) noted that velocity field in GR8 does show a large scale gradient, they chose to characterize the velocity field as giving the overall impression of resulting from random motions.

We present here deep, high velocity resolution $\left(\sim 1.6 \mathrm{~km} \mathrm{~s}^{-1}\right)$ Giant Meterwave Radio Telescope (GMRT) observations of the HI emission from GR8 and use them to study the kinematics of this galaxy. The rest of the paper is divided as follows. The GMRT observations are detailed in Sect. 2, while the results are presented in discussed in Sect. 3. Throughout this paper we take the distance to GR8 to be 2.2 Mpc, and hence its absolute magnitude to be $M_{B} \sim-12.1$.

\section{Observations}

The GMRT observations of GR8 were conducted from 16-18 November 2002. The setup for the observations is given in Table 1. Absolute flux calibration was done using scans on the standard calibrators 3C48 and 3C286, one of which was observed at the start and end of each observing run. Phase calibration was done using 1252+119 which was observed once every $30 \mathrm{~min}$. Bandpass calibration was done in the standard way using $3 \mathrm{C} 286$.

The data were reduced using standard tasks in classic AIPS. For each run, bad visibility points were edited out, after which the data were calibrated. Calibrated data for all runs were combined using DBCON. The GMRT does not do online doppler tracking - any required doppler shifts have to be applied during the offline analysis. However since the differential doppler shift over our observing interval is much less than the channel width, there was no need to apply an offline correction.

The GMRT has a hybrid configuration (Swarup et al. 1991) with 14 of its 30 antennas located in a central compact array with size $\approx 1 \mathrm{~km}(\approx 5 \mathrm{k} \lambda$ at $21 \mathrm{~cm})$ and the remaining antennas distributed in a roughly " $Y$ " shaped configuration, giving a maximum baseline length of $\approx 25 \mathrm{~km}(\approx 120 \mathrm{k} \lambda$ at $21 \mathrm{~cm})$. The baselines obtained from antennas in the central square are similar in length to those of the "D" array of the VLA while the baselines between the arm antennas are comparable in length to the "B" array of the VLA. A single observation with the GMRT hence yields information on both large and small angular scales. Data cubes were therefore made at various $(u, v)$ ranges, including $0-5 \mathrm{k} \lambda, 0-10 \mathrm{k} \lambda, 0-15 \mathrm{k} \lambda$ and $0-80 \mathrm{k} \lambda$ using uniform weighting. At each $(u, v)$ range, a circularly symmetric Gaussian taper with a $F W H M$ equal to $80 \%$ of the $(u, v)$ range was applied, in order to reduce the sidelobes of the synthesized beam. The angular resolutions obtained for the various $(u, v)$ ranges listed above were $40^{\prime \prime} \times 38^{\prime \prime}, 25^{\prime \prime} \times 25^{\prime \prime}, 16^{\prime \prime} \times 14^{\prime \prime}$ and $4^{\prime \prime} \times 3^{\prime \prime}$ respectively. All the three low resolution data cubes (i.e. up to $16^{\prime \prime} \times 14^{\prime \prime}$ resolution) were deconvolved using the the AIPS task IMAGR. For the highest resolution data cube, the signal to noise ratio was too low for CLEAN to work reliably and hence the $4^{\prime \prime} \times 3^{\prime \prime}$ resolution data cube could not be deconvolved. Despite this, the low SNR of this image implies that the inability to deconvolve it does not greatly degrade its dynamic range or fidelity. The morphology of the emission should hence be accurately traced, apart from an uncertainty in the scaling factor (this essentially arises because the main effect of deconvolving weak emission at about the noise level corresponds to multiplying by a scale factor; Jörsäter \& van Moorsel 1995; Rupen 1999).

The HI emission from GR8 spanned 28 channels of the spectral cube. A continuum image was made using the average of remaining line free channels. No continuum was detected from the disk of GR8 to a $3 \sigma$ flux limit of $1.8 \mathrm{mJy} / \mathrm{Bm}$ (for a beam size of $\left.46^{\prime \prime} \times 37^{\prime \prime}\right)$. We also checked for the presence of any compact continuum sources in the disk of GR8 by making a high resolution $\left(6.4^{\prime \prime} \times 5.8^{\prime \prime}\right)$ map - no sources associated with the disk of GR8 were detected down to a $3 \sigma$ limit of $0.6 \mathrm{mJy} / \mathrm{Bm}$.

We examined the line profiles at various locations in the galaxy and found that they were (to zeroth order) symmetric and single peaked. In the very high column density regions, a double Gaussian and/or a gauss-hermite fit does provide a somewhat better description of the data, but even in these regions, the mean velocity produced by the moment method agrees within the errors with the peak velocity of the profile. Since we are interested here mainly in the systematic velocities, moment maps provide an adequate description of the data. Moment maps (i.e. maps of the total integrated flux (moment 0 ), the flux weighted velocity (moment 1 ) and the flux weighted velocity dispersion (moment 2 )) were made from the data cubes using the AIPS task MOMNT. To obtain the moment maps, lines of sight with a low signal to noise ratio were excluded by applying a cutoff at the $3 \sigma$ level, ( $\sigma$ being the rms noise level in a line free channel), after smoothing in velocity (using boxcar smoothing three channels wide) and position 
Table 1. Parameters of the GMRT observations.

\begin{tabular}{ll}
\hline \hline Parameters & Value \\
\hline RA (1950) & $12^{\mathrm{h}} 56^{\mathrm{m}} 10.5^{\mathrm{s}}$ \\
Declination (1950) & $+14^{\circ} 29^{\prime} 17^{\prime \prime}$ \\
Central velocity (heliocentric) & $215.0 \mathrm{~km} \mathrm{~s}^{-1}$ \\
Date of observations & $16-18 \mathrm{Nov} .2002$ \\
Time on source & $16 \mathrm{hrs}$ \\
Total bandwidth & $1.0 \mathrm{MHz}\left(211 \mathrm{~km} \mathrm{~s}^{-1}\right)$ \\
Number of channels & 128 \\
Channel separation & $1.65 \mathrm{~km} \mathrm{~s}^{-1}$ \\
$F W H M$ of synthesized beam & $41^{\prime \prime} \times 39^{\prime \prime}, 25^{\prime \prime} \times 25^{\prime \prime}$, \\
& $4^{\prime \prime} \times 3^{\prime \prime}$ \\
RMS noise per channel & $2.0 \mathrm{mJy}, 1.6 \mathrm{mJy}$, \\
& $1.0 \mathrm{mJy}$ \\
\hline
\end{tabular}

(using a Gaussian with $F W H M \sim 2$ times that of the synthesized beam). Maps of the velocity field and the velocity dispersion were also made in GIPSY using single Gaussian fits to the individual profiles. The velocities produced by MOMNT in AIPS are in reasonable agreement with those obtained using a single Gaussian fit. However the AIPS moment 2 map systematically underestimates the velocity dispersion (as obtained from Gaussian fitting) particularly near the edges where the signal to noise ratio is low. This can be understood as the effect of the thresholding algorithm used by the MOMNT task to identify the regions with signal. From the Gaussian fitting we find that the velocity dispersion $\sigma$ is $\approx 9.0 \mathrm{~km} \mathrm{~s}^{-1}$, and shows only slight variation across the galaxy. This value of $\sigma$ and the lack of substantial variation of $\sigma$ across the galaxy is typical of dwarf galaxies (e.g. Lake et al. 1990; Skillman et al. 1988a).

\section{Results and discussion}

\subsection{HI distribution}

The global HI emission profile of GR8, obtained from $40^{\prime \prime} \times$ $38^{\prime \prime}$ data cube, is shown in Fig. 1. A Gaussian fit to the profile gives a central velocity (heliocentric) of $217 \pm 2 \mathrm{~km} \mathrm{~s}^{-1}$. The integrated flux is $9.0 \pm 0.9 \mathrm{Jy} \mathrm{km} \mathrm{s}{ }^{-1}$. These are in good agreement with the values of $214 \pm 1 \mathrm{~km} \mathrm{~s}^{-1}$ and $8.78 \mathrm{Jy} \mathrm{km} \mathrm{s}^{-1}$ obtained from single dish observations (Huchtmeier et al. 2000). The good agreement between the GMRT flux and the single dish flux shows that no flux was missed because of the missing short spacings in the interferometric observation. The velocity width at the $50 \%$ level $\left(\Delta V_{50}\right)$ is $26 \pm 1 \mathrm{~km} \mathrm{~s}^{-1}$, which again is in good agreement with the $\Delta V_{50}$ value of $27 \mathrm{~km} \mathrm{~s}^{-1}$ determined from the single dish observations. The HI mass obtained from the integrated profile (taking the distance to the galaxy to be $2.2 \mathrm{Mpc}$ ) is $10.3 \pm 1.0 \times 10^{6} M_{\odot}$, and the $M_{\mathrm{HI}} / L_{B}$ ratio is found to be $\sim 1.0$ in solar units.

Figure 2 shows the integrated $\mathrm{HI}$ emission from GR8 at $25^{\prime \prime} \times 25^{\prime \prime}$ resolution, overlayed on the digitized sky survey (DSS) image. The HI distribution is clumpy and shows three major clumps. This is highlighted in Fig. 3 which shows the integrated HI emission at high resolution $\left(4.0^{\prime \prime} \times 3.0^{\prime \prime}\right)$. The faint extended HI gas seen in the low resolution image is resolved out in this image. One may suspect that the diffuse HI emission (particularly that seen in between the three clumps in the low

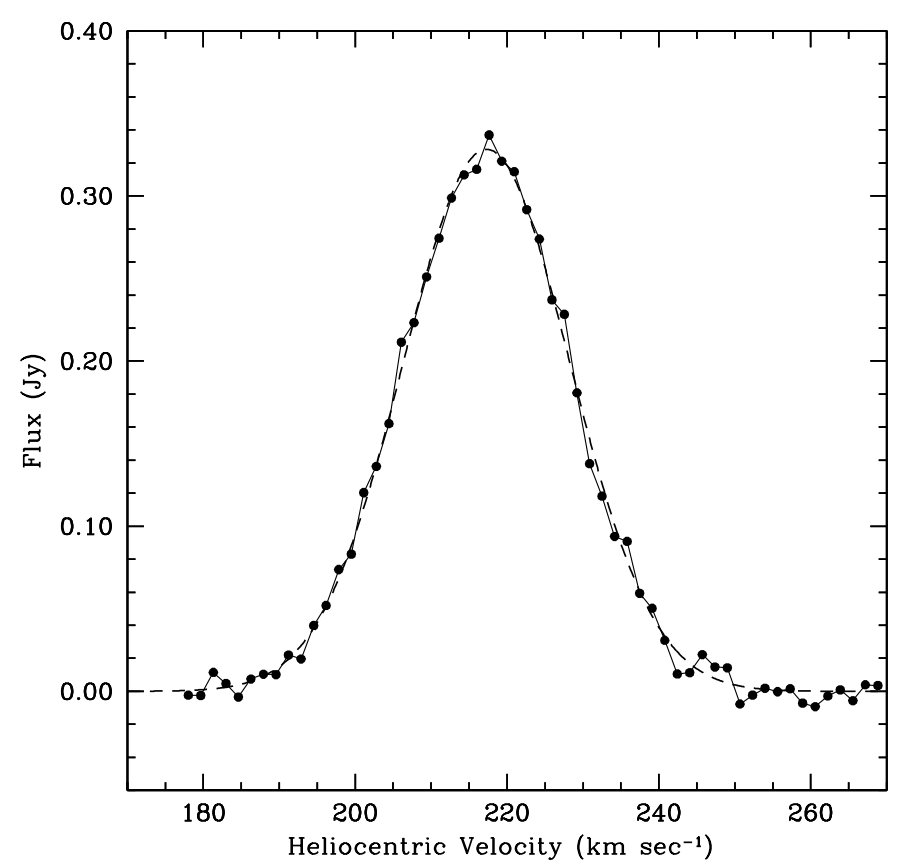

Fig. 1. The integrated spectrum for GR8 obtained from the $40^{\prime \prime} \times 38^{\prime \prime}$ data cube. The channel separation is $1.65 \mathrm{~km} \mathrm{~s}^{-1}$. Integration of the profile gives a flux integral of $9.0 \mathrm{Jy} \mathrm{km} \mathrm{s}^{-1}$ and an HI mass of $\sim 10.3 \times$ $10^{6} M_{\odot}$. The dashed line shows a Gaussian fit to the profile.

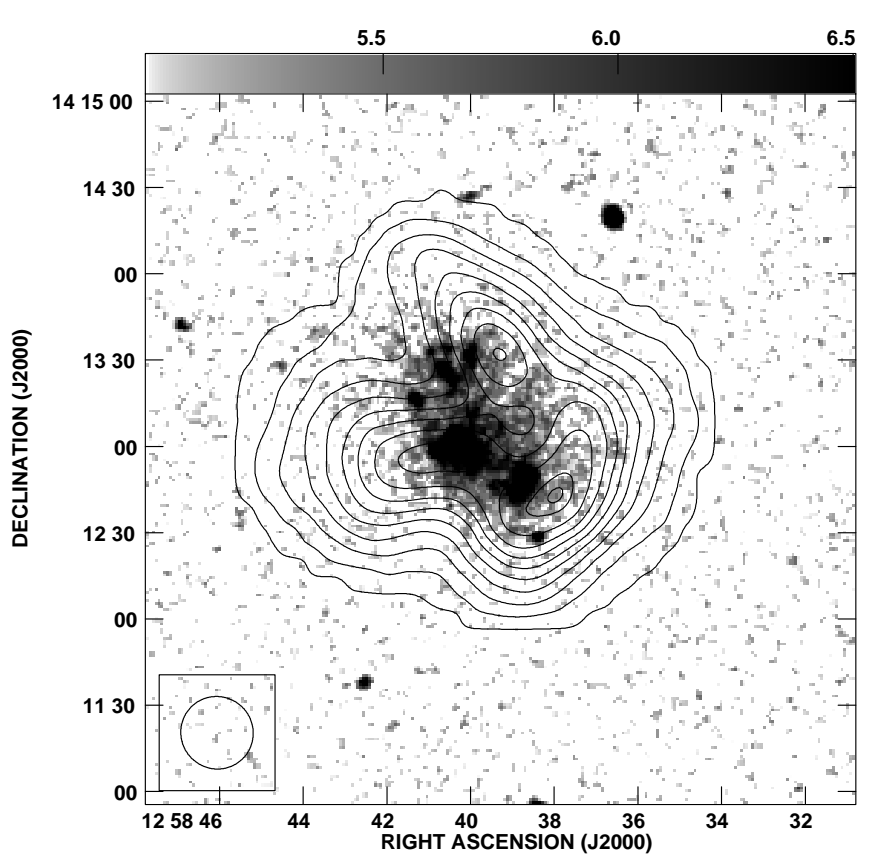

Fig. 2. The digitized Palomar Sky Survey image of GR8 (greyscales) with the GMRT $25^{\prime \prime} \times 25^{\prime \prime}$ resolution integrated HI emission map (contours) overlayed. The contour levels are $0.005,0.076,0.146,0.217$, $0.288,0.359,0.429,0.500,0.571,0.642$ and $0.665 \mathrm{Jy} / \mathrm{Bm} \mathrm{km} \mathrm{s}^{-1}$

resolution map) is not real but is the result of beam smearing. To check for this possibility, the individual channel maps in 


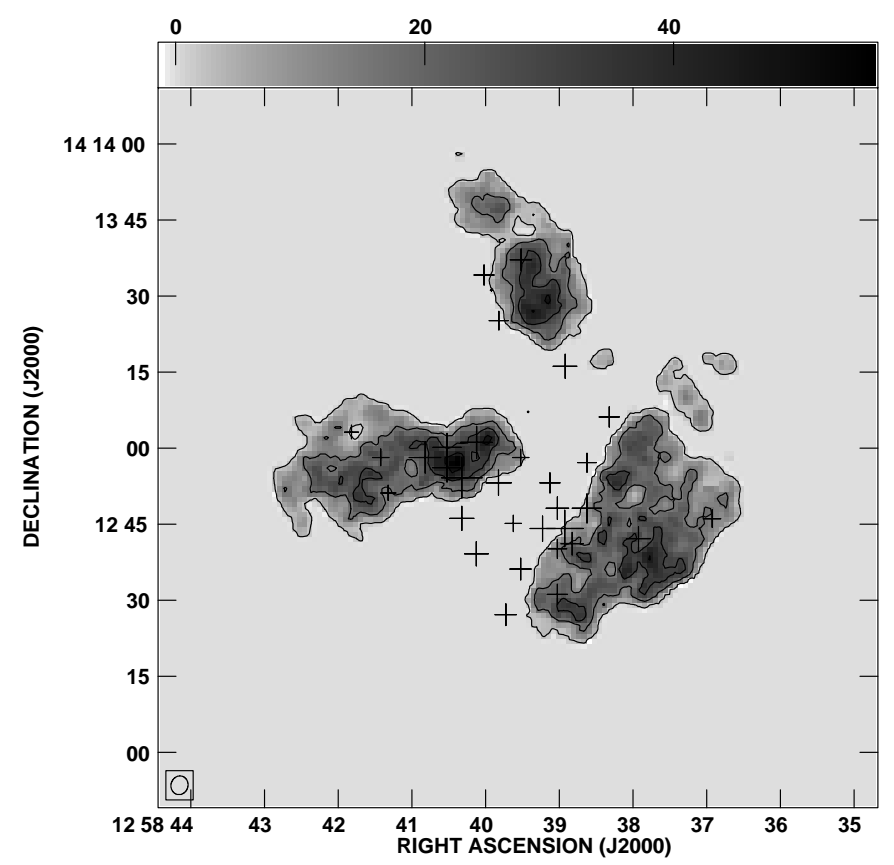

Fig. 3. Integrated $\mathrm{HI}$ emission at $4^{\prime \prime} \times 3^{\prime \prime}$ resolution, (grey scales and contours). The contour levels are 0.002, 0.016, 0.030, 0.044 and $0.058 \mathrm{Jy} / \mathrm{Bm} \mathrm{km} \mathrm{s}^{-1}$. The locations of the HII regions identified by Hodge et al. (1989) are indicated by crosses.

the $25^{\prime \prime} \times 25^{\prime \prime}$ data cube were inspected. In the channel maps, the peak of the diffuse emission in the central region of the galaxy occurs at a different heliocentric velocity than peak velocities of nearby HI clumps, contrary to what one would expect from beam smearing. As a further confirmation of this, the clean components from the $25^{\prime \prime} \times 25^{\prime \prime}$ resolution data cube were convolved with a smaller restoring beam of $10^{\prime \prime} \times 10^{\prime \prime}$, to generate a new data cube. The diffuse emission is visible in the channel maps in this cube, contrary to what would have been expected in case the diffuse emission was entirely due to beam smearing (in which case the clean components would have been restricted to the three clumps).

As can be seen in Fig. 2, each HI clump is associated with a clump of optical emission. However, for each clump, the peak optical emission is generally offset from the peak of the HI emission. The H $\alpha$ image of Hodge et al. (1989) shows that the optical clumps also emit copious amounts of $\mathrm{H} \alpha$ and are hence regions of ongoing star formation. In addition to the bright clumps, diffuse optical emission is also seen in Fig. 2. The optical emission has a much higher ellipticity than the $\mathrm{HI}$ emission and the position angles of the optical and HI major axis can also be seen to be different. Quantitatively, ellipse fitting to the outermost contours of the $40^{\prime \prime} \times 38^{\prime \prime}$ and $25^{\prime \prime} \times 25^{\prime \prime}$ resolution $\mathrm{HI}$ moment maps (which are less distorted by the presence of the HI clumps in the inner regions) gives a position angle of $77 \pm 5$ degrees and an inclination (assuming the intrinsic shape of the HI disk to be circular) of $28 \pm 3$ degrees. The values obtained from the two different resolution maps agree to within the error bars. On the other hand, these values are considerably different from those obtained from ellipse fitting to the optical isophotes, which yields a position angle of 38.4 degrees and an inclination of 57.7 degrees respectively (De Vaucouleurs \& Moss 1983). We return to this issue in Sect. 3.3.

\subsection{HI kinematics}

The velocity field derived from the $25^{\prime \prime} \times 25^{\prime \prime}$ resolution data cube is shown in Fig. 4. This velocity field is in reasonable agreement (albeit of better quality) with that obtained by Carignan et al. (1990). The velocity field shows closed contours and is, to zeroth order, consistent with a velocity field that would be produced by a rotating disk with an approximately north south kinematical major axis. This would make the kinematical major axis roughly perpendicular to the major axis obtained from ellipse fitting to the HI disk. The kinematical major axis is also substantially misaligned with the major axis obtained by ellipse fitting to the optical isophotes. In addition to this misalignment, the kinematical center of the velocity field is offset (to the north, as can be seen by comparing Figs. 4 and 2) from the center (as determined by ellipse fitting) of the HI disk.

Apart from the misalignments mentioned above, the velocity field of GR8 also shows clear departures from what would be expected from an axisymmetric rotating disk. The most important departure is that the isovelocity contours in the outer regions of the galaxy show large scale kinks. In addition, the velocity field shows several asymmetries. The most prominent asymmetry is between the northern and southern half of the galaxy. The closed isovelocity contours in the southern half are more elongated than those in the northern half. Further, the kinks noted above are much more prominent in the western part of the disk than in the eastern half. Since our velocity field is better sampled compared to the velocity fields derived by Lo et al. (1993) and Carignan et al. (1990) these kinematical peculiarities are more clearly seen. In particular, the offset between the morphological and kinematical center, which is apparent in our velocity field is not seen in velocity fields derived earlier. Further, because of the lower sensitivity, the kinks in the isovelocity contours seen towards the edges of the galaxy are not seen that clearly in the earlier velocity fields.

Following Carignan et al. (1990) we could try to fit GR8's velocity field to that expected from a rotating disk. In such a fit one can anticipate (based on the closed isovelocity contours) that the rotation curve would be falling and (based on the kinks in the isovelocity curves on the eastern and western edges of the disk) that either the rotation curve would need to rise again towards the edge of the disk, or the edges of the disk would need to be warped. We discuss rotation and other models for producing the observed velocity field in more detail in the next section.

\subsection{Discussion}

As described in the last two sections, the morphology and kinematics of GR8 are somewhat peculiar. If the HI gas and the stars in GR8 are both in disks, then the stellar disk would have to be both more inclined and have a different position angle than the gas disk. It is more likely that the star formation in 


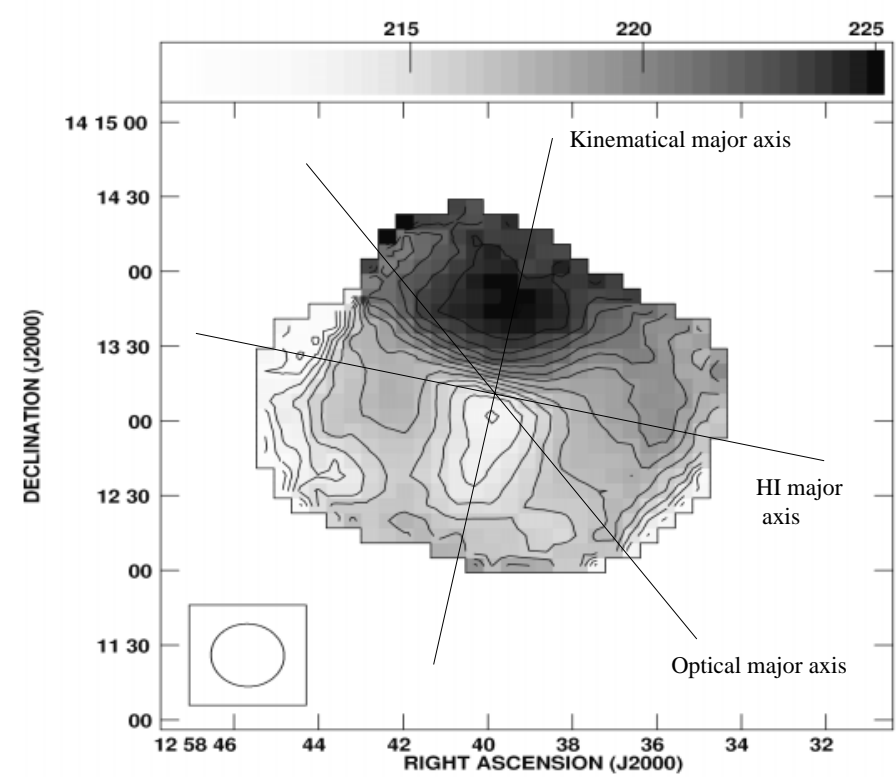

Fig. 4. The HI velocity field of GR8 at $25^{\prime \prime} \times 25^{\prime \prime}$ arcsec resolution. The contours are in steps of $1 \mathrm{~km} \mathrm{~s}^{-1}$ and range from $210.0 \mathrm{~km} \mathrm{~s}^{-1}$ to $225.0 \mathrm{~km} \mathrm{~s}^{-1}$.

GR8 has occurred preferentially in a non axisymmetric symmetric region in the center of the galaxy. In the extreme case, the stars would have a more bar like distribution than the gas. A central stellar bar could affect the gas dynamics, however since the stellar mass is probably not dynamically dominant (from the observed $B-V$ color of 0.38 for GR8 and the low metallicity models of Bell \& de Jong (2001), the the stellar mass is $\sim 5 \times 10^{6} M_{\odot}$, i.e. a factor of 2 less than the HI mass) this effect may not be important.

Apart from having a peculiar morphology, the kinematics of GR8 is also unusual. The kinematical and HI major axis of this galaxy are perpendicular to each other, the kinematical center is offset from the morphological center and the observed velocity field is systematically asymmetric. GR8 is not the only dwarf galaxy which shows misalignment between kinematical and morphological axes, such misalignments have also been seen in, for e.g. Sextans A (Skillman et al. 1988a), NGC 625 (Côté et al. 2000) and DDO 26 (Hunter \& Wilcots 2002). However, the misalignment and off-centered kinematics does imply that GR8 cannot be modeled as a pure axisymmetric rotating disk (for which all axis and centers would be aligned). Although, Carignan et al. (1990) had noted some of these problems, they had nonetheless, modeled the kinematics of GR8 as an axisymmetric rotating disk. Their derived rotation curve had a maximum amplitude of $\sim 8 \mathrm{~km} \mathrm{~s}^{-1}$, and fell sharply with increasing galacto-centric distance.

Our attempts to derive a rotation curve from our velocity field were not successful. The errors in the estimated parameters were large, as were the residuals between the model and the observed velocity field. Our failure to find a good fit (as opposed to Carignan et al. 1990, who were able to fit a rotation curve) is probably related to our better sampling of the velocity field, which, as noted above, makes the misalignments and asymmetries in the velocity field more striking. To provide a feel for the velocity field that would be produced by circular rotation, we show in Fig. 5B the model velocity field that corresponds to the rotation curve of Carignan et al. (1990). The disk has been taken to be intrinsically elliptical (with an axis ratio of $2: 1$ ), so that despite having an inclination of $60^{\circ}$ (the inclination angle derived from the velocity field by Carignan et al. 1990) the projected model HI disk matches the fairly circular appearance of the observed HI disk. Essentially, the foreshortening along the kinematical minor axis is offset by the inherent ellipticity of the disk. As expected, although the model produces closed isovelocity contours along the apparent morphological HI minor axis, the asymmetries seen in the closed contours between northern and southern half of the galaxy, the kinks in the isovelocity contours towards the edges of the disk, as well as the offset between the kinematical and morphological center are not reproduced. As discussed in Sect. 3.2, kinks in the outer isovelocity contours can be produced by requiring the rotation curve to rise again, or by requiring the outer parts of the disk to be extremely warped. Quantitatively, to reproduce the observed kinks, the inclination angle is required to change by an amount sufficient to cause the observed velocity at the edges to increase by a factor of $\sim 2$ compared to the unwarped model. Such extreme warps can, in principle, lead to multiply peaked line profiles. However, because of the low signal to noise ratio towards the edges, we cannot reliably distinguish between single peaked and multiply peaked line profiles in these regions. A more serious concern in modeling the velocity field of GR8 as a rotating disk is the observed misalignment between the kinematical and HI major axes. As noted above, this requires the HI disk to be inherently elongated with an axis ratio of at least $2: 1$. Such a highly non circular disk would be very unusual. Further, the inner regions of the galaxy (i.e. the distance at which the rotation curve of Carignan et al. 1990 peaks) will complete one rotation in $\sim 80 \mathrm{Myr}$, while the rotation period at the edge of the disk is $\sim 1$ Gyr. Hence, this differential rotation will wind up any elongation in the disk on a timescale that is short compared to the age of the galaxy.

Alternatively, as first proposed by Lo et al. (1993), the observed velocity field of GR8 could also be the result of radial motions in the gas i.e. expansion or contraction. Since the sign of the inclination of the galaxy is unknown, it is not possible to distinguish between inward and outward radial motions. Large scale bulk radial gas flows, although difficult to understand in the context of normal spiral galaxies, could nonetheless be plausible in small galaxies like GR8. In models of dwarf galaxy formation and evolution, energy injected into the ISM from stellar winds and supernova explosions could drive significant expansive motions in the gas. In fact, in such models, dwarf galaxies below a critical halo circular velocity of $\sim 100 \mathrm{~km} \mathrm{~s}^{-1}$ are expected to lose a significant fraction of their ISM from the first burst of star formation (e.g. Dekel \& Silk 1986; Efstathiou 2000). Expulsion of the ISM because of the energy input from supernovae is also postulated as a possible mechanism for producing dwarf elliptical galaxies from gas rich progenitors (e.g. Miralda-Escude \& Rees 1997). Observationally, outflows of ionized material have been seen in star bursting dwarf galaxies (e.g. Marlowe et al. 1995). Of course, these models deal with the expulsion of hot supernovae heated gas, where as, in this instance we are dealing with cold neutral gas. For sufficiently 

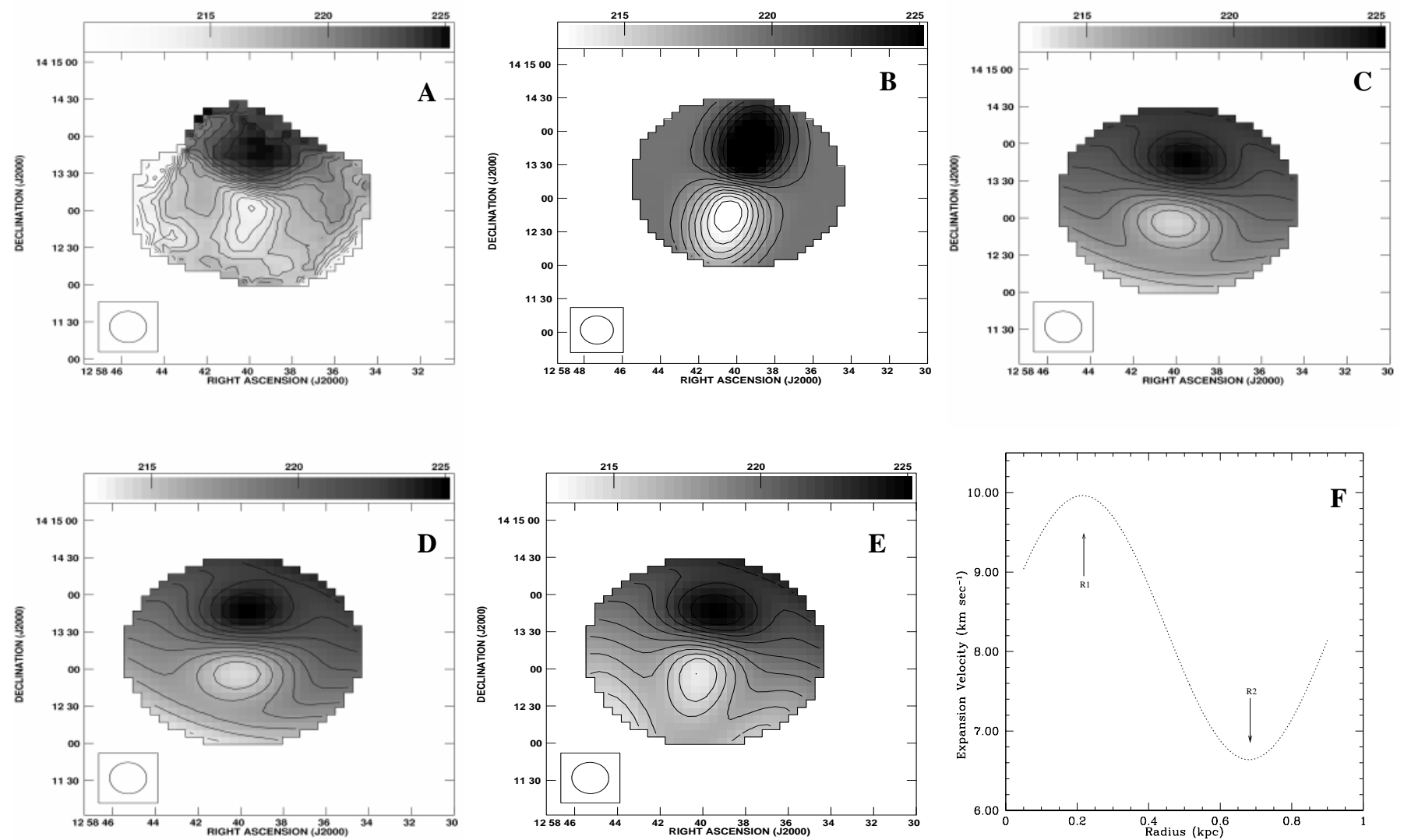

Fig. 5. A) The $25^{\prime \prime} \times 25^{\prime \prime}$ resolution moment 1 map. The contour levels go from 210 to $225 \mathrm{~km} \mathrm{~s}^{-1}$ in steps of $1 \mathrm{~km} \mathrm{~s}^{-1}$. B) The velocity field obtained using the rotation curve of Carignan et al. (1990). Note that we have further assumed that the HI disk is intrinsically elliptical, with an axis ratio of 2:1. See the text for more details. The contours levels in this and succeeding panels are from 213 to $225 \mathrm{~km} \mathrm{~s}^{-1}$ in steps of $1 \mathrm{~km} \mathrm{~s} \mathrm{~s}^{-1}$. C) The model velocity field with only expansion motion in the gas. The expansion velocity used to obtain the model is given in panel F). See also the discussion in the text. D) The model velocity field with both expansion and rotation motions in the gas. The expansion is the same as in panel C). The assumed rotation curve is linear and rises to a maximum of $6 \mathrm{~km} \mathrm{~s}^{-1}$ at the edge of the galaxy. E) The model velocity field with rotational and asymmetric expansion motions. The rotational velocity is the same as used in panel D), however the expansion curve (while similar in form to that in panels C) and D) has been scaled (in galacto-centric distance but not amplitude). F) Expansion curve used to obtain the model velocity field. See the text for more details.

small galaxies however, model calculations (Ferrara \& Tolstoy 2000) suggest that the ISM could be "blown away" i.e. that the ambient medium could be swept out by the hot expanding supernovae superbubbles. This is in contrast to the situation in slightly larger galaxies where there is instead a "blow out" i.e. the supernovae heated hot gas pierces the ambient disk material and escapes into the intergalactic medium. Although a situation where the entire ISM is expanding outwards has not yet been observed, expansion of the neutral ISM on smaller scales has been observed in a number of starbursting dwarf galaxies. Such expanding HI supershells have been seen in, for example, Holmberg II (Puche et al. 1992), IC 2574 (Walter \& Brinks 1999) and Holmberg I (Ott et al. 2001). One should note however, that while the observational evidence for expanding shells in the ISM of these galaxies is reasonably good, the mechanism by which these shells have been created is less well established. Stewart \& Walter (2000) find that the giant supershell in IC 2574 is probably driven by energy input from supernovae, while Rhode et al. (1999), despite deep optical imaging, do not find the star clusters that would be expected to be present in this scenario, at the centers of the HI holes in Holmberg II.
In light of the above discussion, and the ongoing star formation in GR8, it may be reasonable to assume that there are large scale radial flows in the galaxy. If we make this assumption, then the line of sight velocity $V_{\text {los }}$ is related to the circular velocity $V_{\text {rot }}$ and the radial velocity $V_{\text {exp }}$ by the relation

$V_{\text {los }}=V_{\text {sys }}+\left(V_{\text {rot }} \cos (\phi)+V_{\text {exp }} \sin (\phi)\right) \sin (i)$

where $V_{\text {sys }}$ is the systemic velocity, $i$ is the inclination angle, and $\phi$ is the azimuthal angle in the plane of the galaxy $(\phi=0$ along the receding half of the kinematical major axis). The simplest such model is one in which there is no rotation. Figure 5C shows such a model for GR8. In this model the inclination angle of the disk is taken to be $20^{\circ}$ and the position angle $350^{\circ}$. These values were chosen to match the observed velocity field, and are in good agreement with the values expected from the ellipse fitting to the outer HI contours (see Sect. 3.1; note that in the case of radial motion, the velocity gradient is maximum along the morphological minor axis and not the morphological major axis). The expansion is taken to be centered on the kinematical center obtained from the velocity field, and not the morphological center. Since radial motions are probably driven 
by energy from star formation, it is not necessary for the expansion center to be coincident with the geometric center of the HI disk. The expansion $V_{\text {exp }}$ is assumed to be azimuthally symmetric, and its variation with galacto-centric distance is as shown in Fig. 5F. The rise in the expansion velocity till the radius R1 produces the parallel isovelocity contours in the central regions of the galaxy, the fall after $\mathrm{R} 1$ produces the closed contours. The rise in the expansion curve, from radius $\mathrm{R} 2$ onwards, produces the kinks seen in the eastern and western edges of the velocity field. This particular form of expansion was chosen because it provides a good match to the observed velocity field. While it is possible that detailed gas dynamic modeling might be able to reproduce this curve, we have not attempted any such modeling in this paper.

While a pure expansion model does produce the closed contours along the morphological minor axis, it does not produce the asymmetries in the velocity field noted in Sect. 3.2. The next most natural model to try is hence one in which there is also some rotation. A velocity field with the same $V_{\exp }$ as before, but with non zero $V_{\text {rot }}$ is shown in Fig. 5D. The rotation curve has been assumed to be linear; it rises to a maximum of $6 \mathrm{~km} \mathrm{~s}^{-1}$ at the edge of the galaxy. A linearly rising rotation curve was chosen because this form of rotation curve is typical of dwarf galaxies. Other types of rotation curves, i.e. a constant rotation curve, a Brandt curve and an exponential curve (which are seldom observed for dwarf galaxies) were also tried. While a constant rotation curve gives a poor fit to the data, Brandt and exponential curves do not provide a better fit to the observed velocity field than that provided by a linear curve. Since these curves introduce many more free parameters in the model without improving the fit quality they were not explored further. The rotation is assumed to be centered on the morphological center of the galaxy. The inclination and position angle are the same as for the previous model. As can be seen, this does reproduce the asymmetry in the kinks in the isovelocity contours between the eastern and western halves of the galaxy. However, it still does not reproduce the asymmetries in the closed contours. A model which does reproduce most of the features of the observed velocity field is shown in Fig. 5E. This model is similar to that used to produce Fig. 5D, the difference being that the expansion curve is no longer assumed to be azimuthally symmetric. The positions of R1 and R2 in the expansion curve (see Fig. 5F) were allowed to be different at different azimuthal angles in the southern half of the galaxy. However, the maximum amplitude of the expansion curve was taken to be the same in all azimuthal directions. The effect of this was to reproduce the elongated closed contours in the southern half of the galaxy. This asymmetry between the kinematics in the northern and southern halves may be related to the corresponding asymmetry in the distribution of HII regions (see Fig. 3, and also the discussion below). The match can obviously be improved by also allowing an azimuth angle dependent scaling of the amplitude of the expansion curve, but in the absence of a physically motivated prescription for the scaling factor, this would not add much to our understanding of the galaxy's kinematics. It should be noted that it has not been shown that our chosen model provides a unique (or even "best" in some rigorous statistical sense) fit to the observed kinematics of the galaxy. It is possible that one could find different forms for the expansion and rotation curves which also provide adequate fits to the observed velocity field. Strictly speaking, a more robust method would have been to determine a least squares fit to the observed velocity field, allowing for both expansion and rotation. This approach has however not been attempted in this paper.

Figure 5E shows that the observed velocity field of GR8 can be quite well matched by a combination of rotational and expansion motions. Assuming that this interpretation is correct, the natural question that arises is, what drives the expansion of the gas? Energy input from star formation is the obvious suspect. For an expansion velocity of $\sim 10 \mathrm{~km} \mathrm{~s}^{-1}$ and an HI mass of $\sim 10^{7} M_{\odot}$ the corresponding kinetic energy is $\sim 10^{52} \mathrm{erg}$. If we assume that the kinetic energy imparted to the ISM by one supernova explosion is $\sim 10^{51} \mathrm{erg}$ (e.g. Reynolds 1988), this implies that kinetic energy required for the expansion motion is equivalent to the energy output of $\sim 10$ supernovae. It is plausible that this number of supernovae have occurred in GR8 in the recent past. The lack of detection of radio continuum sources (corresponding to the supernovae remnants) would then place a limit on the magnetic field in the galaxy. On the other hand, no star clusters are located at the center of expansion. However, as can be seen from Fig. 3, the majority of the HII regions associated with the three HI clumps lie on the inner edges of the clumps (i.e. towards the center of the galaxy). In a study of HII regions in dwarf galaxies Elmegreen \& Hunter (2000) found that the HII regions tend to have a higher pressure than the average pressure in the disk. They suggest that the HII regions could still be in pressure equilibrium if they preferentially lie in dense HI clumps, where the ambient pressure is higher than the average over the disk. Of the sample of galaxies studied by Elmegreen \& Hunter (2000) GR8 showed the largest pressure anomaly; the pressure in the HII regions was found to be at least a factor of $\sim 55$ times larger than the average disk pressure. Since these HII regions tend to lie at the inner edges of the HI clumps, this over pressure could possibly drive the clumps outwards. It is interesting to note in this regard, that the star formation history of these clumps indicates that they have been forming stars continuously over at least last $500 \mathrm{Myr}$, i.e. the clumps themselves are gravitationally bound (Dohm-Palmer et al. 1998). The measured expansion velocity $\left(\sim 10 \mathrm{~km} \mathrm{~s}^{-1}\right)$ is also considerably smaller than the escape velocity (which would be $\sim 30 \mathrm{~km} \mathrm{~s}^{-1}$, if we assume that GR8 is dark matter dominated and has a dynamic mass to light ratio $\sim 10$ ), which means that the neutral ISM is still bound. This is consistent with the models of dwarf galaxy evolution which include a clumpy ISM - in such models the cold clumpy material does not escape from the galaxy Andersen \& Burkert (2000).

So far we have been treating the radial motions as expansion. Since the sign of the radial motion is unconstrained, we should also note that the velocity field could instead arise from infall. In this case, gas is swirling inwards into the galaxy. The model would then be that GR8 is forming from the merger of the three clumps, and that the diffuse gas and stars are material that have been tidally stripped from the clumps and which are now settling down to form a disk. However, in this scenario, it is unclear if one would obtain the observed velocity field, which doesn't show any clear signature of tidal 
interaction. Another possible infall scenario is that the gas is now falling back towards the center of the potential after a previous expansion phase.

To conclude, we have presented deep, high velocity resolution $\left(\sim 1.6 \mathrm{~km} \mathrm{~s}^{-1}\right)$ GMRT HI $21 \mathrm{~cm}$ synthesis images for the faint $\left(M_{B} \sim-12.1\right)$ dwarf irregular galaxy GR8. We find that though the HI distribution in the galaxy is very clumpy, there is nonetheless substantial diffuse gas. The velocity field of the galaxy is not chaotic, but shows a systematic large scale pattern. We are unable to fit this pattern with either pure rotation or pure expansion. From an inspection of the velocity field however, the following qualitative remarks can be made. If this pattern is treated as arising because of rotation, then (i) the rotation curve would have to be sharply falling, and the disk would have to be extremely warped at the outer edges and (ii) the disk has to inherently elliptical, with an axis ratio $\widetilde{>} 2$. Such a disk would get quickly wound up due to differential rotation. For these reasons we regard it unlikely that GR8's velocity field is due to pure rotation. A more likely model is one in which the kinematics of GR8 can be described as a combination of radial and circular motions. Such a model provides a reasonable fit to the observed velocity field. In this interpretation, in case the radial motions are outwards, then they could be driven by the star formation in GR8; a previous study (Elmegreen \& Hunter 2000) has shown that the pressure in the HII regions in this galaxy is at least 55 times greater than the average pressure in the disk. The measured expansion velocity is considerably less than the estimated escape velocity, so even in this interpretation the cold gas is still bound to the galaxy. Finally, the radial motions could also be interpreted as infall, in which case GR8 is either in the process of formation, or the ISM is falling back after a previous phase of expansion.

Acknowledgements. The observations presented in this paper would not have been possible without the many years of dedicated effort put in by the GMRT staff in order to build the telescope. The GMRT is operated by the National Centre for Radio Astrophysics of the Tata Institute of Fundamental Research. We are grateful to Rajaram Nityananda for many helpful conversations and valuable comments.

\section{References}

Andersen, R. P., \& Burkert, A. 2000, ApJ, 531, 296

Begum, A., Chengalur, J. N., \& Hopp, U. 2003, New A, 8, 267

Carignan, C., Beaulieu, S., \& Freeman, K. C. 1990, ApJ, 99, 178
Côté, S., Carignan, C, \& Freeman, K. C. 2000, ApJ, 120, 3027

Courteau, S., \& van den Bergh, S. 1999, AJ, 118, 337

Dekel, A., \& Silk, J. 1986, ApJ, 303, 39

De Vaucouleurs, G., \& Moss, C. 1983, ApJ, 271, 123

Dohm-Palmer, R. C., Skillman, E. D., Gallagher, J., et al. 1998, ApJ, 116,1227

Efstathiou, G. 2000, MNRAS, 317, 697

Elmegreen, B. G., \& Hunter, D. A. 2000, ApJ, 540, 814

Ferrara, A., \& Tolstoy, E. 2000, MNRAS, 313, 291

Hodge, P. W. 1967, ApJ, 148, 719

Hodge, P., Lee, M. G., \& Kennicutt, R. 1989, PASP, 101, 640

Hoessel, J. G., \& Danielson, D. E. 1983, ApJ, 271, 65

Huchtmeier, W. K., Karachentsev, I. D., Karachentseva, V. E., \& Ehle, M. 2000, A\&A, 141, 469

Hunter, D. A., \& Wilcot, E. M. 2002, AJ, 123, 2449

Jörsäter S., \& van Moorsel, G. A. 1995, AJ, 110, 2037

Ott, J., Walter, F., Brinks, E., et al. 2001, AJ, 122, 3070

Kunth, D., \& Östlin, G. 2000, A\&ARv, 10, 1

Lake, G., Schommer, R. A., \& van Gorkom, J. H. 1990, AJ, 99, 547

Lo, K. Y., Sargent, W. L. W., \& Young, K. 1993, ApJ, 106, 507

Marlowe, A. T., Heckman, T. M., Wyse, R. F. G., \& Schommer, R. 1995, ApJ, 438, 563

Miralda-Escude, J., \& Rees, M. J. 1997, ApJ, 478, 57

Puche, D., Westpfahl, D. Brinks, E., \& Roy, J. R. 1992, AJ, 103, 1841

Reaves, G. 1956, AJ, 61, 69

Reynolds, S. 1988, Galactic and Extragalactic Radio Astronomy, ed. G. Verschuur, \& K. Kellerman (New York: Springer-Verlag), 463

Rhode, K. L., Salzer, J. J., Westpfahl, D. J., \& Radice, L. A. 1999, AJ, 118,323

Rupen, M. P. 1999, Synthesis Imaging in Radio Astronomy II, ASP Conf. Ser., 180, 229

Skillman, E. D., Terlevich, R., Teuben, P. J., \& van Woerden, H. 1988, A\&A, 198, 33

Skillman, E. D., Melnick, J., Terlevich, R., \& Moles, M. 1988, A\&A, 196, 31

Skillman, E. D. 1996, Neutral Hydrogen in Dwarf Galaxies, PASP Conf. Ser., 106, 208

Stewart, S. G., \& Walter, F. 2000, AJ, 120, 1794

Swarup, G., Ananthakrishnan, S., Kapahi, et al. 1991, Current Science, 60, 95

Tolstoy, E., Saha, A., Horssel, J. G., \& Danielson, E. 1995, AJ, 109, 579

van den Bergh, S. 1959, Pub. David Dunlap Obs., 2, 147

van den Bergh S. 2000, PASP, 112, 529

Verter, F., \& Hodge, P. 1995, ApJ, 446, 616

Walter, F., \& Brinks, E. 1999, AJ, 118, 273

Young, L. M., van Zee L., Lo, K. Y., Dohm-Palmer, R. C., \& Beirele M. E. 2003, ApJ, to appear [astro-ph/0304266] 